Available online at:

https://acta-acustica.edpsciences.org

\title{
From Open Access to Open Science: Audio articles and open-source software in Acta Acustica
}

Acta Acustica has launched a new article type called "Audio Articles". Audio Articles are scientific articles with associated audio files. Playable sound clips are embedded in the PDF and HTML versions of the article in the places authors have indicated.

This is an important step for the publication about all subjects in acoustics, including non-audible frequencies. The sounds are now not only depicted in the classical way, i.e., in figures, but also in sound examples. Obviously, this is very useful for music, speech, noise, hearing, etc.

The first audio article is an event for Acta Acustica. It is linked to another paper on the banjo, and the signatures are those of a famous specialist of acoustics and vibration, Jim Woodhouse, Professor at Cambridge University (UK), David Politzer (Caltech, California, winner of the Nobel Prize in Physics (2004), and Hossein Mansour, who is Engineer in San Diego, after a PhD in Mc Gill University (Montreal). Let us cite David Politzer: "As an amateur acoustician, I have for many years been embedding links to sound files in work I post on-line. It certainly makes sense to me". The two papers give a detailed overview of a particular instrument, built with many coupled elements.

Dear Authors, we are waiting your next paper with this new presentation.

To prepare your article, all explanations are given in the instruction for authors. In addition to uploading audio files with your article, you should also upload them in an appropriate open data repository, where they will be available to readers of your article in case they are unable to play the mp3 files embedded in the PDF file. This also ensures that audio files are available for reuse by the wider research community.

In another development, Acta Acustica will soon publish a special issue on "Auditory models: from binaural processing to multimodal cognition". In this special issue, we will present a collection of recent advances in research on conceptual and computational auditory models with a particular focus on spatial hearing. Computational models linked with articles will be collected in the Auditory Modeling Toolbox repository under a free software license, enabling future research in auditory modeling.

Following the relaunch of Acta Acustica in January 2020 as a fully open access journal, these advances contribute to its further evolution towards full "open science", see e.g. the website https://www.ouvrirlascience.fr/open-science/.

Manfred Kaltenbacher, Editor-in-Chief Jean Kergomard, Consulting Editor

Anne Ruimy, Senior Publisher

Cite this article as: Kaltenbacher et al. 2021. From Open Access to Open Science: Audio articles and open-source software in Acta Acustica. Acta Acustica, 5, E1. 